\title{
Comparison of Voice Handicap Index Scores and Treatment Outcomes Between Professional and Non-professional Voice Users with Hoarseness
}

\author{
${ }^{1}$ Surya Sureshkumar, ${ }^{2}$ Unnikrishnan Menon
}

\begin{abstract}
Background: Hoarseness can affect the normal day to day life of people, as the ability to speak is one of the factors which makes us humans superior to other living beings. It is essential for effective communication and reflects one's personality. For some, voice forms the mainstay of their profession. Voice handicap index scores (VHI 30) is a self-assessment questionnaire which evaluates the level of handicap caused due to hoarseness. Patients were divided into groups of professional and non-professional voice users, and after appropriate treatment, the VHI scores were compared between the two. It may be assumed that the professional voice user would fare worse at a certain point of time after treatment.
\end{abstract}

Objective: To compare the VHI 30 score and other voice parameters, and the pathological characteristics of laryngeal lesions between professional and non-professional voice users with hoarseness due to benign laryngeal pathologies, at 6 months posttreatment.

Materials and methods: Evaluation results including the $\mathrm{VHI}$ 30 score were obtained from 46 patients in both the groups (selected as per inclusion-exclusion criteria). These were compared between pretreatment and 6 months posttreatment status. Chi-square test was used to compare between males and females. Independent sample t-test was used to compare the $\mathrm{VHI}$ scores, $\mathrm{VHI}$ change, shimmer, F0, contact quotient (CQ) and age between groups $P$ and N. Mann-Whitney test was used to compare jitter between groups $\mathrm{P}$ and $\mathrm{N}$. Repeated measures analysis of variance (ANOVA) with Bonferroni post hoc correction was used to compare the change in the $\mathrm{VHI}$ score at time points in groups $\mathrm{P}$ and $\mathrm{N}$.

Results: Our study was done in 46 adult patients in each group (professional group-group P and non-professional group-group $\mathrm{N})$. The mean $\mathrm{VHI}$ score pretreatment in group $\mathrm{P}$ was 52.3 and 25.6 after 6 months of treatment. In group $\mathrm{N}$, the pretreatment value was 52.1 and posttreatment was 22.3. Comparing the mean difference of $\mathrm{VHI}$ score between the two groups showed no statistically significant difference ( $p$-value 0.319 ). Comparison of rest of the voice parameters also showed no statistically significant difference. Maximum phonation duration (MPD)

\footnotetext{
${ }^{1}$ Junior Resident, ${ }^{2}$ Associate Professor

1,2Department of Otorhinolaryngology, Amrita Institute of Medical Sciences Kochi, Kerala, India
}

Corresponding Author: Unnikrishnan Menon, Associate Professor, Department of Otorhinolaryngology, Amrita Institute of Medical Sciences Kochi, Kerala, India, e-mail: unnikrishnanmenon8@ gmail.com p-value 0.319; Jitter p-value 0.173, Shimmer p-value 0.313, F0 $p$-value $0.893, C Q$-value 0.225$)$. Vocal nodule was found to be having higher incidence $(65.2 \%$ and $60.9 \%$ in groups $P$ and $\mathrm{N}$ respectively), followed by vocal cord polyp (23.9\% and $17.4 \%$ in sroups $P$ and $N$ respectively), and vocal fold cyst (10.9\% and $21.7 \%$ in groups $P$ and $N$ respectively) without an statistically significant difference between both the groups ( $p$-value 0.314 )

Conclusion: There is no statistically significant difference in posttreatment VHI score, other voice parameters and the characteristics of laryngeal pathologies between the two groups, but the non-professional group showed more improvement numerically irrespective of the nature of treatment availed.

Keywords: Hoarseness, Microlaryngeal surgery, Professional voice user, Voice handicap index (VHI) 30.

How to cite this article: Sureshkumar S, Menon U. Comparison of Voice Handicap Index Scores and Treatment Outcomes Between Professional and Non-professional Voice Users with Hoarseness. Int J Phonosurg Laryngol 2018;8(1):1-5.

Source of support: Nil

Conflict of interest: None

\section{INTRODUCTION}

Voice is a quality that helps humans in the art of speech, which makes them superior to other living beings. The ability to speak is an important aspect in our day to day life. There are many who have conquered the world, and people's minds, with the mere means of speaking. Conversely, there are those unfortunate ones who have to suffer the consequences of various voice or speech disabilities.

Of these disabilities, the commonest symptom is described by the term 'hoarseness,' which can be explained as a perceived rough, harsh or breathy quality to the voice. ${ }^{1}$ It can be of varied etiology, including benign or malignant neoplasms. However, a majority of voice disorders is caused by wrong or inappropriate use of this faculty. Amongst these, individual voice requirement at work is often the culprit. The present study has focused on this aspect viz. the professional voice user, as against the non-professional voice user, suffering from the symptom of hoarseness.

Evaluation and thereby treatment of laryngeal pathologies, have evolved over the years because of the 
better understanding of the physiology of phonation as well as ever-improving hardware and software. Manuel Garcia first observed the movement of his own vocal cords using a laryngeal mirror and sunlight as the light source. ${ }^{2}$ Since then, many techniques for demonstrating laryngeal anatomy and function have been developed. Primary level involves the use of the indirect laryngoscopy mirror or the rigid angled scope.

These gave way to the use of trans-nasal flexible fiberoptic endoscopy in the 1980s. Currently, the modality of choice is videolaryngostroboscopy (VLSS), which helps in literally "throwing light on the voice." Parameters such as mucosal wave over the vibrating vocal folds have revolutionized the assessment of various vocal cord pathologies causing hoarseness. The other aspect has been the creation of soft-wares to analyze voice and speech characteristics. The present study utilizes Dr Speech. Thirdly, subjective assessment using the VHI 30 is also an essential component.

As regards treatment, surgical modality is restricted to cysts and polyps on the vocal folds. Voice strain spectrum disorders, including vocal nodules, are treated by voice therapy. The latter form the majority of cases of hoarseness. In either case, the further prognosis depends, to a large extent, on the voice care measures inculcated by the patient in his/her lifestyle, after the appropriate treatment.

This study aims to compare the treatment outcomes of professional and non-professional voice users having hoarseness caused by benign vocal fold lesions, up to 6 months posttreatment, using VHI 30 as the primary tool.

\section{OBJECTIVE}

To compare the VHI 30 score and other voice parameters, and the pathological characteristics of laryngeal lesions between professional and nonprofessional voice users with hoarseness due to benign laryngeal pathologies, at 6 months post treatment.

\section{MATERIALS AND METHODS}

\section{Selection and Description of Participants}

A prospective comparison study was conducted on 46 patients categorized into groups of professional voice user (group P) and non-professional voice user (group N), according to the nature of their profession, fulfilling both inclusion and exclusion criteria.

\section{Inclusion Criteria}

Those in the age group of 18 years and above, presenting with voice disorder, and found to have benign vocal pathology.

\section{Exclusion Criteria}

- Patients below the age of 18 years

- Those with the malignant glottic lesion

\section{Technical Information}

The study was conducted at Amrita Institute of Medical Sciences, Kochi, Kerala, India with the following objectives:

\section{Primary Objective}

To compare the VHI 30 between professional and nonprofessional voice users with hoarseness because of benign laryngeal lesions at 6 months post-treatment

\section{Secondary Objectives}

- To compare other voice analysis parameters between professional and non-professional voice users with hoarseness due to benign laryngeal lesions at 6 months post-treatment

- To compare the pathological characteristics of the laryngeal lesions in both groups

Relevant history was taken. Each patient was administered with the VHI 30 questionnaire and was scored. The examination was done, which included checking the MPD, followed by laryngeal examination (mirror and/or rigid scope).The pati ent was then subjected to VLSS. All the relevant details were entered into the voice proforma in the hospital system. Each patient was referred to the Department of Audiology and Speech Pathology, for detailed computerized analysis of voice. Following this, each patient underwent the appropriate treatment (voice therapy/micro-laryngeal surgery). They were followed up in voice clinic after one month and six months posttreatment and evaluated with VHI, VLSS and acoustic analysis, and the findings were documented.

Study duration: 18 months

\section{Statistics}

Since no similar study could be located with the primary aim to compare the VHI 30 score between professional and non-professional voice users with hoarseness posttreatment, a pilot study was conducted on 10 patients in each group. Based on the outcome mean and standard deviation, with $95 \%$ confidence and $80 \%$ power, minimum sample size came to a minimum of 44 in each group. We have included 46 patients in each group in this study. Chi-square test was used to compare between males and females. Independent sample t-test was used to analyze the VHI scores, VHI change, Shimmer, F0, 
contact quotient (CQ) and age between groups $\mathrm{P}$ and $\mathrm{N}$. Mann Whitney test was used to compare Jitter between groups $\mathrm{P}$ and N. Repeated measures analysis of variance (ANOVA) with Bonferroni post hoc correction was used to compare the change in the VHI score at time points in groups $\mathrm{P}$ and $\mathrm{N}$. The analysis was performed using software version Statistical Package for the Social Sciences (SPSS) 20.0 for Windows.

\section{RESULTS}

In the present study, the mean age group in groups $\mathrm{P}$ and $\mathrm{N}$ was 43.3 and 43.8 respectively. It was found to have a female preponderance in both the groups, with 32 (69.6\%) females in group P and 28 (60.9\%) female in group $\mathrm{N}$. The baseline characteristics of the study population is furnished in Table 1.

Microlaryngeal surgery (MLS) was performed in cases of vocal fold polyps and cysts, while nodules resolved with voice therapy. Among 46 patients in each group, 13 patients (28.3\%) in group P required MLS and the rest $33(71.7 \%)$, voice therapy. In group $\mathrm{N}$, the corresponding numbers were $16(34.8 \%)$ and $30(65.2 \%)$ respectively. As expected, there was a significant improvement in both the groups irrespective of the nature of treatment provided, which was demonstrated by laryngostroboscopy and on comparing the mean VHI score and other acoustic parameters pre-treatment and 1 and 6 months post-treatment.

The mean VHI score pre-treatment in group P was 52.3, which has improved to 37.5 one month post-treatment, and to 25.6 after 6 months of treatment. In group $\mathrm{N}$, the mean VHI pre-treatment was 52.1. One month posttreatment, it was found to have decreased to 38.6, and at 6 months, it was 22.3. On comparing the mean VHI change from the pre-treatment period to 6 months posttreatment, in group $\mathrm{P}$ the mean change was 26.7 , and in group $\mathrm{N}$ it was 29.8. There was no statistically significant difference between the two groups. But numerically more improvement was shown by group $\mathrm{N}$ (Table 2 ).

Comparison of the acoustic parameters like MPD, jitter, shimmer, fundamental frequency and CQ showed improvement in both groups, without any statistically significant difference. The mean MPD in group $P$ pretreatment was 7.2 , which was increased to

Table 1: Baseline characteristics of the study population

\begin{tabular}{lllll}
\hline & \multicolumn{2}{l}{ Group $P(n=46)$} & Group N $(n=46)$ \\
\cline { 2 - 5 } Characteristics & Mean & $S D$ & Mean & Mean \\
\hline Age (Years) & 43.3 & 11.2 & 43.8 & 14.9 \\
& Female & Male & Female & Male \\
Gender & $\mathrm{n} \%$ & $\mathrm{n} \%$ & $\mathrm{n} \%$ & $\mathrm{n} \%$ \\
& $32(69.6)$ & 14 & $28(60.9)$ & $18(39.1)$ \\
& & $(30.4)$ & & \\
\hline
\end{tabular}

Table 2: Mean difference in $\mathrm{VHI}$ in groups $\mathrm{P}$ and $\mathrm{N}$; VHI 6-mean difference between VHI value at the time of the first visit and at the time of 6 months posttreatment

\begin{tabular}{|c|c|c|c|c|c|}
\hline \multirow{2}{*}{$\begin{array}{l}\text { VHI } \\
\text { change }\end{array}$} & \multicolumn{2}{|c|}{$\begin{array}{l}\text { Group P } \\
n=46\end{array}$} & \multicolumn{2}{|c|}{$\begin{array}{l}\text { Group N } \\
n=46\end{array}$} & \multirow[b]{2}{*}{$p$-value } \\
\hline & Mean & $S D$ & Mean & $S D$ & \\
\hline VHI 6 & 26.7 & 16.3 & 29.8 & 14.1 & 0.319 \\
\hline
\end{tabular}

10.4 six months posttreatment. The same in group $\mathrm{N}$ was 6.9 pretreatment, improving to 10.8 at six months posttreatment. In the case of jitter, the mean pretreatment value was 0.27 in group $\mathrm{P}$, and 0.16 at six-month posttreatment. In group $\mathrm{N}, 0.24$ was the pretreatment value, and 0.14 the same at six months posttreatment. In group $\mathrm{P}$, the mean Shimmer was 1.68 pretreatment, which has come down to 1.28 at six months posttreatment. The same in group $\mathrm{N}$, pretreatment value was 1.60 , and posttreatment value was 1.24 Fundamental frequency, mean value in the pretreatment period was found to be 167.6 and six months post-treatment value was 197.0 in group P. In group $\mathrm{N}$, the pretreatment value was 170.8 , and six months post-treatment, it was 197.9. In group $\mathrm{P}$, the mean cq was 49.9 in the pretreatment period, and 75.4 at six month posttreatment. In group $\mathrm{N}$, it was 45.5 , pretreatment, and 71.4 six months, posttreatment (Table 3).

On analyzing the characteristics of the benign vocal fold lesions, vocal nodule was found to be having higher incidence $(65.2 \%$ and $60.9 \%$ in groups $\mathrm{P}$ and $\mathrm{N}$ respectively), followed by vocal cord polyp (23.9\% and $17.4 \%$ in groups $\mathrm{P}$ and $\mathrm{N}$ respectively), and vocal fold cyst (10.9\%

Table 3: Comparison of other voice parameters between both groups at first visit (MPD, jitter, shimmer, FO, CQ), one month post-treatment (MPD 1, jitter 1, shimmer 11, FO 1, CQ 1), six month post-treatment (MPD 6, jitter 6, shimmer 6, FO 6, CQ 6)

\begin{tabular}{|c|c|c|c|c|c|}
\hline & \multicolumn{2}{|c|}{ Group P $n=46$} & \multicolumn{2}{|c|}{ Group $N n=46$} & \multirow[b]{2}{*}{$p$-value } \\
\hline & Mean & SD & Mean & SD & \\
\hline MPD & 7.2 & 2.5 & 6.9 & 1.7 & 0.386 \\
\hline MPD 1 & 9.3 & 2.1 & 8.8 & 2.2 & 0.268 \\
\hline MPD 6 & 10.4 & 2.3 & 10.8 & 2.0 & 0.385 \\
\hline Jitter & 0.27 & 031 & 0.24 & 0.28 & 0.695 \\
\hline Jitter 1 & 0.23 & 0.27 & 0.21 & 022 & 0.900 \\
\hline Jitter 6 & 0.16 & 0.08 & 0.14 & 0.03 & 0.173 \\
\hline Shimmer & 1.68 & 0.36 & 1.60 & 0.33 & 0.256 \\
\hline Shimmer 1 & 1.46 & 0.26 & 1.42 & 0.25 & 0.433 \\
\hline Shimmer 6 & 1.28 & 0.18 & 1,24 & 0.16 & 0.313 \\
\hline FO & 167.6 & 42.9 & 170.5 & 43.2 & 0.720 \\
\hline F0 1 & 186.2 & 37,2 & 153.0 & 39.7 & 0.692 \\
\hline TO 6 & 197. & 0318 & 197,9 & 34.4 & 0,893 \\
\hline $\mathrm{CQ}$ & 49.9 & 20.3 & 45.5 & 20.0 & 0.291 \\
\hline CQ 1 & 64.0 & 16.7 & 58.5 & 20.3 & 0.162 \\
\hline CQ 6 & 75.4 & 13.0 & 71.4 & 18.3 & 0.225 \\
\hline
\end{tabular}


Table 4: Comparison of various benign pathologies between both groups

\begin{tabular}{llll}
\hline & $\begin{array}{l}\text { Group } \\
P\end{array}$ & $\begin{array}{l}\text { Group } N \\
n(\%)\end{array}$ & $p$-value \\
\hline Pathology & $n(\%)$ & $10(21.7)$ & 0.314 \\
Vocal fold cyst & $5(10.9)$ & $11.4)$ & \\
Vocal fold polyp & 11 & $8(17.4)$ & \\
& $(23.9)$ & & \\
Vocal fold nodule & 30 & $28(60.9)$ & \\
& $(65.2)$ & & \\
\hline
\end{tabular}

and $21.7 \%$ in groups $\mathrm{P}$ and $\mathrm{N}$ respectively). But there was no statistically significant difference found between both the groups in the nature of incidence of the pathological lesions (Table 4).

\section{DISCUSSION}

The vocal fold has a complex histological structure. The phenomenon, known as "cover-body structure" is at the core of this. ${ }^{3}$ It is the mainstay of vocal fold vibration during the act of phonation. This is explained as the passive movement of the vocal fold cover over its body as air flows through the glottis. It follows that any pathology leading to alteration of either the cover or the body of the vocal fold can affect its vibration. Benign lesions like polyps, cysts, and nodules are typical examples. Of these, vocal nodules are more commonly encountered. People who are professionally dependent on their voice are found to be more prone to develop hoarseness, as they are unintentionally forced to make excessive use of their voice.

Several studies have been conducted on the effect of hoarseness and the treatment outcome on professional voice users, and in the majority, teachers were considered. In the study conducted by Martins et al. (Brazil), ${ }^{4}$ there is a 2 to 3 fold more incidence of voice disorders in teachers than in the general population.

In the present study, the mean age group in groups $\mathrm{P}$ and $\mathrm{N}$ was 43.3 and 43.8 respectively. Many studies conducted worldwide had shown a higher incidence of hoarseness due to benign vocal fold lesions in females. One such study was that of Hunter et al. ${ }^{5}$ In our study, a similar scenario was obtained with $32(69.6 \%)$ females in group P and $28(60.9 \%)$ in group $\mathrm{N}$.

It was demonstrated by Dikkers et al. ${ }^{6}$ in their study of benign lesions of the vocal fold by histopathology that, the most common benign lesions are polyps, followed by nodules, cysts and Reinke's edema. In contrast to this, our study showed a higher incidence of vocal fold nodules in both the groups in comparison to vocal polyps and cysts, which in turn was comparatively more in number in the professional group (group P) than the non-professional one (group N). But there was no statistically significant difference found between both the groups in the nature of incidence of the pathological lesions.

Treatment is provided to restore the normal vibratory function of the vocal fold, thereby providing relief from dysphonia. The modality of treatment can be either conservative (voice therapy) or surgical (MLS for excision), according to nature and severity of the lesion.

Posttreatment, objective and subjective evaluation of laryngeal functions are done periodically to assess the treatment progress. Laryngeal evaluation by laryngoscopy helps in assessing the nature of the lesion affecting vocal fold function. The same also helps in monitoring the post-treatment status. Stroboscopy has been an important evaluation methodology in this study in visualizing and comparing the mucosal wave patterns of the vocal fold in various pathologies, and also the change with treatment. Several articles have been cited proposing the efficacy of stroboscopy in the field of laryngology. One such is that of Mehta et al. ${ }^{7}$

Acoustic measures evaluated in the Department of Audiology and Speech pathology using Dr Speech software have also played an important role in this study, by demonstrating the changes of the same with various lesions, and also post-treatment. This was as suggested by the protocol proposed by Dejonckere et al. ${ }^{8}$ Comparison of the acoustic parameters like MPD, jitter, shimmer, fundamental frequency and contact quotient showed improvement in both groups, without any statistically significant difference. As expected, there was a significant improvement in both the groups irrespective of the nature of treatment provided, which was demonstrated by laryngostroboscopy and on comparing the mean VHI score and other acoustic parameters pre-treatment and 1 and 6 months posttreatment.

The VHI 30 has been found to be a useful entity, by acting as a self-assessment tool for the patients, thereby helping in evaluating the functional, emotional and physical impact of hoarseness in their day-to-day life, and also in monitoring the treatment outcome in the patient's own viewpoint. There are several studies which prove the same, one of them being conducted by Maertens et al. ${ }^{9}$ Similar to the results demonstrated by Spina et al. ${ }^{10}$ it was found that there is a significant impact of hoarseness on the daily activities and profession of a patient. Even though there was a significant improvement in both the groups posttreatment, which was demonstrated by the laryngoscopic and acoustic parameter evaluation, this difference in mean VHI score between both the groups may be because of the fact that, the treatment outcome may not have attained the level of expectation of the professional 
group, as their livelihood is dependent on their voice. In contrast to this finding, the interpretation by Cheng et al. showed a statistically significant improvement in their professional group. ${ }^{11}$

The aim of this prospective study was to see whether the treatment outcome would be different between professional and non-professional voice users, over a certain duration. This was based on the hypothesis that a professional voice user would return to his/her vocal overuse pattern, leading to reduced treatment. Although intuitively this would seem to be the case, there have been no definitive studies of this nature. We have attempted to follow-up these two group of patients, in a systematic way, up to a duration of 6 months, posttreatment.

\section{STUDY LIMITATIONS}

Only three types of benign pathological lesions were included (vocal fold nodule, polyps, and cysts). Professional group was not further subclassified. Inadequate number of patients to get a statistically significant result, given the time frame of the study

\section{CONCLUSION}

The VHI 30 scores between professional and non-professional voice users at 6 months posttreatment showed no statistically significant difference. However, numerically the non-professional group had more improvement. The comparison of rest of the voice analysis parameters showed improvement in both groups 6 months posttreatment, without any statistically significant difference. The evaluation of pathological characteristics of the benign vocal cord lesions between both groups showed a higher incidence of vocal nodules, followed by vocal fold polyp and cyst, in that order, without any statistically significant difference. Significant improvement was shown by patients of both the groups, which was as expected, irrespective of the nature of treatment obtained.

\section{CLINICAL SIGNIFICANCE}

Hoarseness is a common symptom in the field of laryngology, often underestimated in many cases. This study, hopefully, can be a portal to assess the quality of life of patients with hoarseness, especially those who are professionally dependant on their voice.

\section{REFERENCES}

1. Gleeson MJ, Clarke RC, editors. Scott-Brown's Otorhinolaryngology: Head and Neck Surgery $7 \mathrm{Ed}$ : 3 volume set. CRC Press; 2008 Apr 25.

2. Garcia M. Observations on the human voice. Proceedings of the Royal Society of London. 1856 Jan 1;7:399-410.

3. Hirano M. Morphological structure of the vocal cord as a vibrator and its variations. Folia Phoniatrica et Logopaedica. 1974;26(2):89-94

4. Martins RH, Pereira ER, Hidalgo CB, Tavares EL. Voice disorders in teachers. A review. Journal of Voice. 2014;28(6): 716-724.

5. Hunter EJ, Tanner K, Smith ME. Gender differences affecting vocal health of women in vo-cally demanding careers. Logopedics Phoniatrics Vocology. 2011 Oct 1;36(3):128-136.

6. Dikkers FG, Nikkels PG. Benign lesions of the vocal folds: histopathology and phonotrauma. Annals of Otology, Rhinology \& Laryngology. 1995 Sep;104 (9):698-703.

7. Mehta DD, Hillman RE. Current role of stroboscopy in laryngeal imaging. Current opinion in otolaryngology \& head and neck surgery. 2012;20(6):429.

8. Dejonckere PH, Bradley P, Clemente P, Cornut G, CrevierBuchman L, Friedrich G, et al. A basic protocol for functional assessment of voice pathology, especially for investigating the efficacy of (phonosurgical) treatments and evalu-ating new assessment techniques. European Archives of Oto-rhinolaryngology. 2001 Feb 8;258(2):77-82.

9. Maertens K, de Jong FI. The voice handicap index as a tool for assessment of the biopsychosocial impact of voice problems. B-ent. 2007;3(2):61-66.

10. Spina AL, Maunsell R, Sandalo K, Gusmão R, Crespo A. Correlation between voice and life quality and occupation. Brazilian journal of otorhinolaryngology. 2009 Apr 30;75(2):275-279.

11. Cheng J. Correlation between the Voice Handicap Index and voice laboratory measurements after phonosurgery. Ear, Nose \& Throat Journal. 2010 Apr 1;89(4):183. 